\title{
Qubit-assisted thermometry of a quantum harmonic oscillator
}

\author{
Matteo Brunelli, ${ }^{1}$ Stefano Olivares, ${ }^{1,2}$ Mauro Paternostro, ${ }^{3}$ and Matteo G. A. Paris ${ }^{1,2}$ \\ ${ }^{I}$ Dipartimento di Fisica, Università degli Studi di Milano, I-20133 Milano, Italy \\ ${ }^{2}$ CNISM, UdR Milano, I-20133 Milano, Italy \\ ${ }^{3}$ Centre for Theoretical Atomic, Molecular and Optical Physics, \\ School of Mathematics and Physics, Queen's University Belfast, Belfast BT7 1NN, United Kingdom
}

(Dated: June 18, 2021)

\begin{abstract}
We use the theory of quantum estimation in two different qubit-boson coupling models to demonstrate that the temperature of a quantum harmonic oscillator can be estimated with high precision by quantum-limited measurements on the qubit. The two models that we address embody situations of current physical interest due to their connection with ongoing experimental efforts on the control of mesoscopic dynamics. We show that population measurements performed over the qubit probe are near optimal for a broad range of temperatures of the harmonic oscillator.

PACS numbers: 42.50.-p, 03.65.-w
\end{abstract}

\section{INTRODUCTION}

The improved control over systems of intrinsic complexity makes the implementation of techniques for the inference of specific properties of their states a necessary step towards the achievement of full quantum control. Yet, it is often the case that the device into which we would like to enforce quantum mechanical features is not fully or easily addressable. System-interrogation can only be performed, in such cases, in an indirect way through the use of probes of an appropriate nature [1]. It is thus very important to devise experimentally implementable strategies for the inference of properties of inaccessible quantum systems, identify the optimal statepreparation of the probe as well as the observable that allows for the maximum extraction of information about the parameter that we are interested in.

This agenda is dressed of even more relevance due to the recent experimental efforts produced towards the quantumlimited management of mesoscopic systems, such as superconducting devices [2], light-interfaced cold-atom systems [3] and mechanical systems operating at the quantum level [4]. All such systems have in common the use of "quantum interfaces" with devices of a different nature, which are then exploited for state-preparation, manipulation of information and possibly read-out. The quantum-interface paradigm is indeed very fruitful for the extraction of information out of a system that is only partially accessible: through the coupling with a controllable subsystem, one can indeed arrange for mechanisms able to provide useful knowledge on key features of a dynamics or a state. Examples of such a possibility, which have been materialised in successful experimental demonstrations, include the micro-maser technology for the revelation of the properties of the field within a high-quality microwave cavity [5], the coupling of a Bose-Einstein condensate to a (classical) mechanical oscillator for the investigation on Casimir-Polder effects [6], and intra-cavity quantum optomechanics, where the radiation-pressure force is used to read the noise properties of a mechanical mode [7]. In the latter context, in particular, a key parameter is embodied by the temperature at which the mechanical mode operates. Indeed, unwanted thermal effects typically spoil the quantum features enforces in the mechanical system by means of a coherent evolution. Having a precise quantitative estimate of the entity of such effects [8] would be crucial not only for prediction purposes but also to design in the best possible way a quantum-enforcing protocol that accounts, ab initio, such undesired effects. Needless to say, these considerations can be extended straightforwardly to any of the scenarios addressed above.

Recently, strategies for the determination of the temperature of a harmonic oscillator have been put forward, based on the coupling to a quantum probe embodied by a two-level system (a qubit) [9, 10]. The coupling model to be sued for the thermometry of the oscillator's state was the JaynesCummings one, within and beyond the so-called rotating wave approximation [11]. While Ref. [10] proposed the use of the ac Stark effect as a way to infer the temperature of the oscillator, Brunelli et al. [9] have applied the proper tools of quantum estimation theory (QET) [12-14] to design optimal protocols for the estimate of the system's temperature.

In this paper, we significantly extend the approach in [9] to other physically motivated qubit-oscillator models, proving that optimal and effective thermometry can indeed be performed by means of simple measurements onto the qubit's state. We tackle both the coupling between a superconducting qubit and a nano electromechanical oscillator and the far-off resonant interaction between a two-level atom and the field of a cavity, thus providing an analytical QET-based study of an ample spectrum of experimentally motivated situations.

The remainder of this work is organized as follows: in Sec. [I] we describe the general system that we address and introduce the QET tools for our analysis. Sec. III studies the first model of our investigation, which addresses the capacitive coupling of a superconducting qubit and a nanoelectromechanical oscillator. In Sec. [V] we assess our QETbased approach in the case of a qubit that is off-resonantly coupled to a harmonic oscillator, such as for a two-level atom in a far-off resonant cavity. In both instances, population measurements over the probing qubit allow for the optimized estimate of the oscillator's temperature. Finally, in Sec. V we summarize our findings and open up new perspectives. 


\section{APPROACH TO THE PROBLEM}

Let us consider a general quantum harmonic oscillator with frequency $\Omega$ and at thermal equilibrium with its environment. The state of the oscillator is described by the Gibbs density operator (we use natural units, i.e. $\hbar=1$, throughout the manuscript)

$$
\rho_{o}=\frac{e^{-\beta \Omega a^{\dagger} a}}{\mathcal{Z}}=\sum_{n=0}^{\infty} \frac{\bar{n}^{n}}{(\bar{n}+1)^{n+1}}|n\rangle\langle n|,
$$

where $\bar{n}=\left(e^{\beta \Omega}-1\right)^{-1}$ is the average number of thermal excitations, $|n\rangle$ is a Fock state with $n$ quanta, $\mathcal{Z}=\operatorname{Tr}\left[e^{-\beta \Omega a^{\dagger} a}\right]$ is the partition function and $\hat{a}\left(\hat{a}^{\dagger}\right)$ is the bosonic annihilation (creation) operator of the harmonic oscillator. Our aim is to estimate the inverse temperature $\beta=1 / k_{B} T$ of the oscillator by coupling it with a qubit encoded in the logical states $\left\{|0\rangle_{q},|1\rangle_{q}\right\}$ of a two-level system that is initially prepared in the general pure state

$$
|\psi\rangle_{q}=\cos \frac{\theta}{2}|0\rangle+e^{i \varphi} \sin \frac{\theta}{2}|1\rangle .
$$

Here $(\theta, \varphi)$ are the angles defining the orientation of the qubit's Bloch vector in the corresponding Bloch sphere, while $k_{B}$ is the Boltzmann constant. We assume no initial correlation between the probe and the oscillator and also assume that the interaction Hamiltonian has the general form

$$
\hat{H}_{I}=g \hat{A}_{o} \otimes \hat{A}_{q},
$$

where $\hat{A}_{o}\left(\hat{A}_{q}\right)$ is an operator in the Hilbert space of the oscillator (qubit) and $g$ a coupling constant. In what follows, we shall call $\left\{|x\rangle_{o}\right\}$ a basis of states of the harmonic oscillators that are eigenstates of $\hat{A}_{o}$, i.e. $\hat{A}_{o}|x\rangle_{o}=x|x\rangle_{o}$.

Any measurement aimed at estimating the temperature of the oscillator is performed on the state $\varrho_{q}$ of the probing qubit after its joint evolution with system $o$. That is

$$
\begin{aligned}
\varrho_{q}(\beta) & =\operatorname{Tr}_{o}\left[\hat{U}|\psi\rangle_{q q}\langle\psi| \otimes \rho_{o} \hat{U}^{\dagger}\right] \\
& =\int d x \rho_{o}(x) e^{-i g t x \hat{A}_{q}}|\psi\rangle_{q q}\langle\psi| e^{i g t x \hat{A}_{q}}
\end{aligned}
$$

where $\rho_{o}(x)={ }_{o}\left\langle x\left|\rho_{o}\right| x\right\rangle_{o}$ are the diagonal matrix elements of the initial thermal state in the basis $|x\rangle_{o}$ of the oscillator operator $\hat{A}_{o}$. In what follows, we make use of the apparatus of QET to design the optimal probing state and measurements needed to estimate the inverse temperature $\beta$. According to the Cramér-Rao inequality, the variance $\delta(\gamma)$ of any unbiased estimator of an arbitrary quantity $\gamma$ satisfies the inequality

$$
\delta(\beta) \geq \frac{1}{M F(\beta)}
$$

with $M$ the number of measurements used in order to perform the estimate and $F(\beta)$ the Fisher information of $\beta$, which is defined as

$$
F(\beta)=\sum_{j} p_{j}\left(\partial_{\beta} \ln p_{j}\right)^{2}=\sum_{j} \frac{\left|\partial_{\beta} p_{j}\right|^{2}}{p_{j}},
$$

where $p_{j}$ is the probabilities to get outcome $j$ from a measurements performed over the qubit state and described, in general, by the positive operator valued measurement (POVM) $\left\{\hat{\Pi}_{j}: \hat{\Pi}_{j} \geq 0, \sum_{j} \hat{\Pi}_{i}=\mathbb{1}\right\}$. Such probabilities are calculated assuming the oscillator at the inverse temperature $\beta$, i.e. $p_{j}=\operatorname{Tr}_{q}\left[\varrho_{q}(\beta) \hat{\Pi}_{j}\right]$.

The quantum mechanical counterpart of the Fisher Information is defined as

$$
H(\beta)=\operatorname{Tr}\left[\varrho_{q} \hat{L}^{2}(\beta)\right]
$$

with $\hat{L}(\beta)$ the symmetric logarithmic derivative operator, satisfying the equation

$$
\partial_{\beta} \varrho_{q}=\left[\hat{L}(\beta) \varrho_{q}+\varrho_{q} \hat{L}(\beta)\right] / 2
$$

The quantum Fisher Information (QFI) is an upper bound for $F(\beta)$ as it embodies the optimization of the Fisher Information over any possible measurement performed over the probing qubit states. The QFI is thus independent of the specific measurement strategy and is an intrinsic feature of the family of probing states. Eq. (4) can then be rewritten as

$$
\delta(\beta) \geq \frac{1}{M H(\beta)},
$$

which extends the Cramér-Rao bound to the quantum domain and embodies the ultimate limit to the precision of the estimate of $\beta$. A measurement is optimal when the corresponding Fisher information $F(\beta)$ equals the quantum Fisher Information $H(\beta)$. Although various instances of optimal measurement may be found, depending on the model at hand, the observable embodied by the spectral measure of $\hat{L}(\beta)$ is certainly optimal. Upon diagonalization of the probe state $\varrho_{q}=\varrho_{+}\left|\psi_{+}\right\rangle\left\langle\left.\psi_{+}\right|_{q}+\varrho_{-} \mid \psi_{-}\right\rangle\left\langle\left.\psi_{-}\right|_{q}\right.$, the QFI can be computed explicitly as

$$
\begin{aligned}
H(\beta)= & \sum_{k= \pm} \frac{\left(\partial_{\beta} \varrho_{k}\right)^{2}}{\varrho_{k}} \\
& +2 \gamma \sum_{k \neq l= \pm}\left|\sum_{j=0,1}\left(\partial_{\beta}\left\langle j \mid \psi_{k}\right\rangle\right)\left\langle\psi_{l} \mid j\right\rangle\right|^{2}
\end{aligned}
$$

with $\gamma=\left(1-2 \varrho_{+}\right)^{2}$ [9]. In what follows, we consider two exactly solvable models corresponding to interesting physical situations and compute the QFI to assess the ultimate precision in the estimation of temperature achievable by any measurements performed on the states of the probing system. We will compare such optimal performance to what is obtained through the Fisher Information associated with population measurements of the probe, i.e. for $\left\{\hat{\Pi}_{j}\right\}=$ $\left\{|0\rangle\left\langle\left. 0\right|_{q}, \mid 1\right\rangle\left\langle\left. 1\right|_{q}\right\}\right.$. We show that in some cases, population measurements are indeed optimal for the estimation of temperature.

\section{JAYNES-CUMMINGS COUPLING BEYOND THE ROTATING WAVE APPROXIMATION}

The first model that we address corresponds to the choice $\hat{A}_{o} \equiv \hat{X}_{o}=\left(\hat{a}+\hat{a}^{\dagger}\right) / \sqrt{2}$, i.e. the in-phase quadrature op- 
erator of the harmonic oscillator, and $\hat{A}_{q}=\hat{\sigma}_{x}$, which is the $x$-Pauli spin operator. Correspondingly, the interaction reads

$$
\hat{H}_{I}=g \hat{X}_{o} \otimes \hat{\sigma}_{x} .
$$

This model is encountered in a few different contexts. On one hand, it describes the effective interaction Hamiltonian for the electric-dipole coupling between a two-level atom and the field of a cavity, thus embodying the celebrated JaynesCummings Hamiltonian [11] beyond the so-called rotating wave approximation. Moreover and rather less intuitively, the same model is achieved by considering a nanomechanical oscillator (a nano beam) coupled capacitively to a Cooperpair box (CPB) operating at the so-called charge degeneracy point [16], where the dynamics of the CPB can be righteously be approximated to that of a two-level system encoded in the space spanned by states $| \pm\rangle=(|0\rangle \pm|1\rangle) / \sqrt{2}$. Here $\{|0\rangle,|1\rangle\}$ are states with exactly 0 and 1 excess Cooper pairs in the large superconducting island shown in Fig. 1. The natural Hamiltonian of the system reads

$$
\hat{H}_{1}=\frac{\left(\hat{Q}-Q_{g}\right)^{2}}{2 C_{t}}-E_{J} \cos \hat{\phi}+\Omega \hat{a}^{\dagger} \hat{a}
$$

with $\hat{Q}$ and $\hat{\phi}$ the canonical charge and phase operator of the $\mathrm{CPB}, C_{t}$ the total capacitance of the island, $Q_{g}=C_{g} V_{g}+$ $C_{x} V_{x}$ the total gate charge, $E_{J}$ the Josephson energy and $\Omega$ the frequency of the nanomechanical oscillator, as indicated above [16]. By defining $\hat{\sigma}_{x}=|+\rangle\langle-|+|-\rangle\langle+|$, expanding $\hat{H}_{1}$ in series of the ratio $x / d$ between the actual position of the mechanical oscillator and its equilibrium distance from the CPB (the amplitude of the oscillations is assumed to be small enough that only terms proportional to $x / d$ are retained in such expansion) and adjusting the gate and driving voltages



FIG. 1: (Color online) An electrically driven nanomechanical oscillator (bias voltage $V_{x}$ ) is coupled to a CPB through the capacitance $C_{x}$. The state of the CPB is controlled by the gate voltage $V_{g}$ (coupled to the box through the capacitance $C_{g}$ ) and the Josephson energy $E_{J}$. We work at the charge degeneracy point. such that $Q_{g} \simeq 0$, the interaction Hamiltonian of the system can be cast into the form

$$
\hat{H}_{1}=\lambda\left(\hat{a}+\hat{a}^{\dagger}\right) \otimes \hat{\sigma}_{x} \equiv g \hat{X} \otimes \hat{\sigma}_{x}
$$

with $\lambda=g / \sqrt{2}$ an effective coupling rate whose form is inessential for our tasks.

The estimate of the temperature in this particular context is especially relevant. Indeed, the nano beam is in contact with a thermal phononic background due to the substrate onto which it is nano-fabricated [17]. The coupling with the superconducting qubit addressed above holds the potential to prepare non-classical states of the nano-beam. Indeed, the time-evolution operator corresponding to Eq. (11) reads, in the qubit basis, as

$$
\hat{U}(t)=\cos (g t \hat{X}) \mathbb{1}_{q}-i \sin (g t \hat{X}) \hat{\sigma}_{x} .
$$

Let us assume that the nano-beam is initialized in a coherent state $|\alpha\rangle(\alpha \in \mathbb{R})$, while the qubit is prepared in $|+\rangle_{q}$. The evolution will generate the qubit-oscillator state

$$
|\eta(\alpha)\rangle_{q o}=\frac{1}{\sqrt{2}}\left(|\alpha+i g t\rangle_{o}|0\rangle_{q}+|\alpha-i g t\rangle_{o}|1\rangle_{q}\right) .
$$

As $|\langle\alpha-i g t \mid \alpha+i g t\rangle|^{2}=e^{-4 g^{2} t^{2}}$, for $g t \simeq \pi$ the two coherent states $|\alpha \pm i g t\rangle$ are quasi-orthogonal and Eq. 12 is almost maximally entangled. By projecting the qubit onto $|+\rangle_{q}$, we achieve the coherent-state superposition $\mathcal{N}\left(|\alpha+i g t\rangle_{o}+\right.$ $\left.|\alpha-i g t\rangle_{o}\right)(\mathcal{N}$ is a normalization factor) which embodies, in the limit of quasi-orthogonal coherent states mentioned above, a highly non-classical state. However, a thermal-state preparation of the harmonic oscillator will smear out such nonclassicality, pushing the state towards the statistical mixture

$$
\rho_{o, t h}=\int d^{2} \alpha G(\alpha, V)|\eta(\alpha)\rangle_{o o}\langle\eta(\alpha)|
$$

where $G(\alpha, V)$ is a Gaussian distribution of width $V=$ $2 \bar{n}+1$ [18]. Determining the exact initial temperature of the nano beam is thus key for the success of such conditional strategies for the enforcement of non-classical features. Our approach to the estimate of $\beta$ will follow the general strategy described above, which we now describe for the specific model in Eq. (11).

The elements of the state of the probing qubit after the interaction with the oscillator and the trace over its degrees of freedom can be calculated explicitly as

$\varrho_{q}(\beta)=\frac{1}{2}\left[\begin{array}{cc}1+\cos \theta e^{-\zeta} & \sin \theta\left(\cos \varphi-i \sin \varphi e^{-\zeta}\right) \\ \sin \theta\left(\cos \varphi+i \sin \varphi e^{-\zeta}\right) & 1-\cos \theta e^{-\zeta}\end{array}\right]$

with $\zeta=\operatorname{coth}\left(\frac{\beta}{2}\right) \tau^{2}$, where $\beta=\frac{\Omega}{k_{B} T}$ and $\tau=g t$ are respectively the dimensionless inverse temperature and interaction time. The Fisher Information associated with a measurement of the populations of $\varrho_{q}(\beta)$, i.e. a measurement of the $z$-Pauli operator $\hat{\sigma}_{z}$, reads

$$
F(\beta)=\frac{\cos ^{2} \theta \operatorname{csch}^{4}(\beta / 2)}{e^{2 \zeta}-\cos ^{2} \theta} \frac{\tau^{4}}{4} .
$$


which is a function of $\beta, \theta, \tau$. Compared to the case where the rotating wave approximation is invoked [9], i.e. for a qubitoscillator interaction of the form $g\left(\hat{a}^{\dagger} \hat{\sigma}_{-}+h . c\right.$. $)$ with $\hat{\sigma}_{ \pm}$the ladder operators of the qubit, the Fisher Information displays a symmetric behavior with respect to $\theta$ and is no longer a periodic function of the time $\tau$. The maximum is achieved by choosing $\theta=\{0, \pi\}$, i.e. by preparing the qubit either in $|0\rangle$ or $|1\rangle$, while for $\theta=\frac{\pi}{2}$ the Fisher Information identically vanishes. Finally, upon choosing one of the optimal qubit preparations, we found that the maximum value of the Fisher Information only depends on temperature. In fact, the value of the dimensionless time at which the Fisher Information is maximized is the following function of $\beta$

$$
\begin{aligned}
\tau_{\text {opt }}(\beta) & =\sqrt{\left[1+\frac{1}{2} W\left(-\frac{2}{e^{2}}\right)\right] \tanh \left(\frac{\beta}{2}\right)} \\
& \simeq 0.893 \times\left(1-e^{-\beta}\right)
\end{aligned}
$$

with $W(y)$ the Lambert function of argument $y$ [20]. The behavior of $\tau_{o p t}(\beta)$ is shown in the left panel of Fig. 2. while in the right one we give examples of the form of the $F(\beta)$ for large temperatures. Remarkably, in the relevant regime $\beta \gg 1$ (low temperatures), the optimal interaction time becomes almost independent on $\beta$. This means that no fine tuning of the interaction time is needed and only a rough a priori information is needed to implement the optimal measurement.

In order to evaluate the quantum Fisher Information, we have diagonalized the state of the probe, as described in Sec. II The explicit calculation, which produces expressions too involved to be reported here, shows that $H(\beta)$ is maximized for two independent sets of choices of the qubit-state parameters. One can either prepare the qubit in one of the basis states $|0\rangle$ or $|1\rangle$, independently of the angle $\varphi$, or choose $\varphi=\{\pi / 2,3 \pi / 2\}$, regardless of $\theta$ (cf. Fig. 3). The values of the QFI are the same in both cases, and the analytic expression of $H(\beta)$ reduces to the one taken by $F(\beta)$ for the choice $\theta=0$. This demonstrates that population measurements are optimal for the whole range of temperatures.

This conclusion is further strengthened by the analysis of the spectral measure of the symmetric logarithmic derivative $\hat{L}(\beta)$. For both the optimal probe-state preparations, $\hat{L}(\beta)$ is



FIG. 2: (Color online) Left: The functional form of $\tau_{\text {opt }}(\beta)$. Right: $F(\beta=3)$ against $\theta$ and $\tau$. Clearly, the Fisher Information is optimized at $\theta=0, \pi$ and quickly decays as $\tau$ grows.

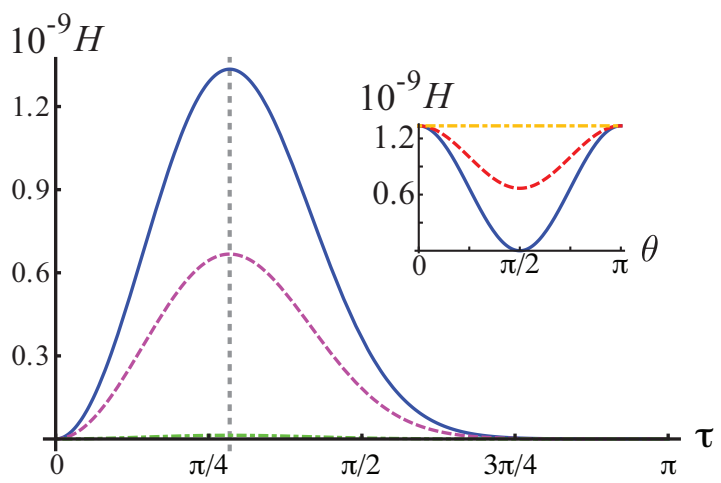

FIG. 3: (Color online) Temporal evolution of the quantum Fisher Information for $\beta=10, \varphi=0$ and $\theta=0$ (blue), $\theta=\frac{\pi}{4}$ (magenta), $\theta=\frac{\pi}{2}+0.1$ (green). For $\theta=0$, the quantum Fisher Information is maximum (independently on $\varphi$ ) and equals the Fisher Information associated to population measurements, while for $\theta=\frac{\pi}{2} H(\beta)$ identically vanishes. Inset: the quantum Fisher Information evaluated at the optimal time, plotted against $\theta$ and for $\varphi=0$ (blue), $\varphi=\frac{\pi}{4}$ (red), $\varphi=\frac{\pi}{2}$ (orange). The last choice leads to the same maximum value of the quantum Fisher Information and does not depend on $\theta$.

diagonal and reads

$$
L(\beta)=-\frac{\tau^{2}}{4} \operatorname{csch}^{2}\left(\frac{\beta}{2}\right)\left[(\operatorname{coth} \zeta-1) \hat{\mathbb{1}}+\operatorname{csch} \zeta \hat{\sigma}_{z}\right] .
$$

with $\hat{\sigma}_{z}$ the $z$-Pauli operator. The explicit presence of such operator in Eq. (16) demonstrates the optimality of population measurements for the estimation of temperature in this model.

We end the section by noticing that if the harmonic oscillator is moved away from the equilibrium position, i.e. its initial state is described by the displaced thermal state $D(\alpha) \varrho_{o} D^{\dagger}(\varrho), D(\alpha)=\exp \left\{\alpha a^{\dagger}-\bar{\alpha} a\right\}$ being the displacement operator, then the probe qubit after the interaction is given by

$$
\varrho_{q}(\alpha, \beta)=e^{-i g \alpha \sigma_{x} \tau} \varrho_{q}(\beta) e^{i g \alpha \sigma_{x} \tau},
$$

where $\varrho_{q}(\beta)$ is the probe state of Eq. (14). The QFI is equal to the zero displacement case, whereas the Fisher information is in general smaller than for zero displacement.

\section{FAR OFF-RESONANT SPIN-BOSON INTERACTION}

We now address a second qubit-oscillator interaction model, specified by taking $\hat{A}_{o}=\hat{a}^{\dagger} \hat{a}$ and $\hat{A}_{q}=\hat{\sigma}_{x}$. The interaction Hamiltonian thus becomes

$$
\hat{H}_{2}=g \hat{a}^{\dagger} \hat{a} \otimes \hat{\sigma}_{x}
$$

This model describes for a two-level system interacting far off-resonantly with a bosonic mode. Let us consider a twolevel system (bosonic mode) with transition frequency $\omega(\Omega)$, interacting through a Jaynes-Cummings model with strength $\lambda$. We call $\Delta=\Omega-\omega$ the detuning between the two systems. 
The corresponding time-evolution operator can be written, in the basis $\{|1\rangle,|0\rangle\}$ of the two-level system, as [15]

$$
\hat{U}=\left[\begin{array}{cc}
\cos \left(\hat{\Omega}_{n+1} t\right)-\frac{i \Delta}{2} \hat{K}_{1+n} & -i \lambda \hat{a} \hat{K}_{n} \\
i \lambda \hat{a}^{\dagger} \hat{K}_{n} & \cos \left(\hat{\Omega}_{n} t\right)+\frac{i \Delta}{2} \hat{K}_{n}
\end{array}\right]
$$

where

$$
\hat{K}_{n}=\frac{\sin \left(\hat{\Omega}_{n} t\right)}{\hat{\Omega}_{n}},
$$

$\hat{\Omega}_{n}=\sqrt{\frac{1}{4} \Delta^{2}+\lambda^{2} \hat{a}^{\dagger} \hat{a}}$ is the effective Rabi frequency operator, and $\hat{a}\left(\hat{a}^{\dagger}\right)$ are the field operators of the boson. For $\Delta^{2} / 4 \gg \lambda^{2}\left\langle\hat{a}^{\dagger} \hat{a}\right\rangle$, we have

$$
\begin{aligned}
& \hat{U}_{10}=\hat{U}_{01} \simeq 0 \\
& \hat{U}_{11} \simeq e^{-i \hat{\Omega}_{n+1} t} \quad \hat{U}_{00} \simeq e^{i \hat{\Omega}_{n} t},
\end{aligned}
$$

and by moving to a reference frame rotating at frequency $\Delta$, we gain the effective picture [19]

$$
\hat{U} \simeq e^{-i \frac{\lambda^{2}}{\Delta} \hat{a} \hat{a}^{\dagger} t}|1\rangle\left\langle 1\left|+e^{i \frac{\lambda^{2}}{\Delta} \hat{a}^{\dagger} \hat{a} t}\right| 0\right\rangle\langle 0| .
$$

We can now shift the energy of the two-level system so that $|g\rangle$ becomes the zero-energy state and

$$
\hat{U}=e^{-i \hat{H}_{2}^{\prime} t}=e^{-i \frac{\lambda^{2}}{\Delta} \hat{a} \hat{a}^{\dagger}|1\rangle\langle 1| t},
$$

with $\hat{H}_{2}^{\prime}$ the interaction Hamiltonian between the boson and the two-level system. By reminding that $|1\rangle\langle 1|=2 \hat{\sigma}_{z}+\mathbb{1}$ and neglecting an inessential term depending only on the qubits' degrees of freedom, we gather the non-trivial interaction term $\frac{2 \lambda^{2}}{\Delta} \hat{a}^{\dagger} \hat{a} \otimes \hat{\sigma}_{z}$. This is locally equivalent (via a Hadamard gate applied to the two-level system) to the model $\hat{H}_{2}$ in Eq. (17). We now assume this interaction model for the probe-oscillator dynamics and the protocol for the estimate of the temperature.

The matrix elements of the probe state after evolution and the trace over the oscillator are

$$
\begin{aligned}
& \varrho_{q, 00}=\cos ^{2} \frac{\theta}{2}+\Gamma\left[2 \zeta \cos \theta \operatorname{sinc}^{2} \tau-\sin \theta \sin \varphi \sin (2 \tau)\right], \\
& \varrho_{q, 01}=\frac{\sin \theta \cos \varphi}{2}+i \Gamma\left\{\left[e^{\beta}-\cos (2 \tau)\right] \sin \theta \sin \varphi-\cos \theta \sin (2 \tau)\right\}
\end{aligned}
$$

with $\varrho_{q, 10}=\varrho_{q, 01}^{*}, \varrho_{q, 11}=1-\varrho_{q, 00}$ and

$$
\Gamma=\frac{1-e^{-\beta}}{4[\cos (2 \tau)-\cosh \beta]} .
$$

The expression taken by the Fisher Information is, in this case, too lengthy to be reported. Qualitatively, $F(\beta)$ depends on both $\theta$ and $\varphi$ and, as in the Jaynes-Cummings model under the rotating-wave approximation, is a periodic function of time $\tau$. The probe state preparation that optimises the Fisher Information is again $\theta=\{0, \pi\}$. For both such choices, $F(\beta)$ is independent of $\varphi$. However, as soon as the qubit initialization deviates from the optimal cases, $F(\beta)$ suddenly drops by several orders of magnitude, as shown in the left panel of Fig. 4. displaying a weak dependence on $\varphi$ [taking $\varphi=\frac{\pi}{2}$ gives the maximum of $F(\beta)$, see the right panel of Fig. 4]. However, the values attained by the Fisher Information in at such optimal values of $\varphi$ are negligible with respect to those associated to $\theta=\{0, \pi\}$ [there is a difference of four orders of magnitude between the values in the left and the right panels of Fig. 4], which makes $\theta$ the only effective qubit parameter.


FIG. 4: (Color online) Left: FI for $\beta=10, \varphi=\frac{\pi}{2}$ as a function of the effective time $\tau$, for different $\theta$ values: $\theta=0$ (blue), $\theta=0.01$ (magenta) and $\theta=0.1$ (green). For $\theta=0$ the Fisher Information does not depend on $\varphi$. Right: Fisher Information for $\beta=10$ and $\theta=\frac{\pi}{4}$ as a function of time for different $\varphi: \varphi=\frac{\pi}{2}$ (blue), $\varphi=\frac{\pi}{3}$ (magenta) and $\varphi=\frac{\pi}{6}$ (green). Although the choice $\varphi=\frac{\pi}{2}$ maximizes the Fisher Information, it is evident that the relevant parameter in setting the qubit is $\theta$.

If we prepare the qubit in an eigenstate of $\hat{\sigma}_{z}$, the Fisher Information associated to a population measurement reads

$$
F(\beta)_{\text {opt }}=\frac{2 e^{2 \beta} \sin ^{2} \tau\left[1+\sinh \beta-\cos (2 \tau) e^{-\beta}\right]^{2} \tanh \left(\frac{\beta}{2}\right)}{\left(e^{\beta}-1\right)\left[1+\left(e^{\beta}-\cos (2 \tau)\right)\left(2 e^{\beta}-1\right)-e^{\beta} \cos (2 \tau)\right][\cos (2 \tau)-\cosh \beta]^{2}} \stackrel{\beta \gg 1}{\simeq} e^{-\beta} \sin ^{2} \tau
$$

On the other hand, by inspecting $H(\beta)$ we found, as before, a symmetric behavior with respect to the qubit parameters: at a given dimensionless time $\tau, H(\beta)$ is maximum either for $\theta=\{0, \pi\}$ (regardless of $\varphi$ ), or $\varphi=\left\{\frac{\pi}{2}, \frac{3}{2} \pi\right\}$ (regardless of $\theta$ ) and the values achieved by the QFI are equal in both cases. Hence the effective contribution to the dynamics comes from 
one octant of the Bloch sphere. The analytic expression of the optimal quantum Fisher Information is

$$
\begin{aligned}
H_{\text {opt }}(\beta) & =\frac{\sin ^{2} \tau\left[2 \cos (2 \tau)-2 \cosh \beta-\sinh ^{2} \beta\right]}{2[\cos (2 \tau)-\cosh \beta]^{3}} \\
& \stackrel{\gg 1}{\simeq} e^{-\beta} \sin ^{2} \tau .
\end{aligned}
$$

The crucial point here is that, for this model, the optimal Fisher Information and its quantum mechanical counterpart are no longer the same, i.e. population measurements are not the optimal one for the whole set of parameters: at low temperatures, provided that we choose an optimal qubit-state preparation, we retrieve the optimality of $\hat{\sigma}_{z}$ measurements as $F_{\text {opt }}(\beta)=H_{\text {opt }}(\beta)$. When the temperature is raised, on the other hand, small discrepancies appear between the temporal behavior of these quantities, suggesting that $\hat{\sigma}_{z}$ is not longer the best measurement strategy. This is shown in Fig. 5, where we study the relative difference between optimized Fisher and quantum Fisher Information.

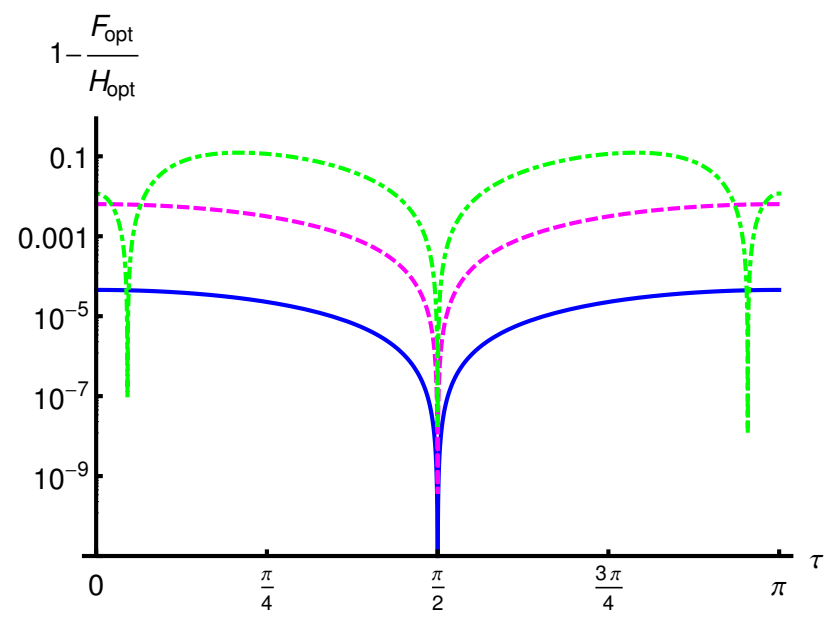

FIG. 5: (Color online) Logarithmic plot of the quantity $\left[H_{\text {opt }}(\beta)-F_{\text {opt }}(\beta)\right] / H_{\text {opt }}(\beta)$ for $\beta=10$ (blue), $\beta=5$ (magenta) and $\beta=1$ (green).

Some insight comes from the analysis of the symmetric logarithmic derivative, which reads

$$
L(\beta)=L_{0} \hat{\mathbb{1}}-L_{x} \hat{\sigma}_{z}+L_{z} \hat{\sigma}_{z}
$$

showing the presence of a contribution coming from a term proportional to $\hat{\sigma}_{x}$, which is responsible for the differences between the two estimators. The expression of the coefficients reads as follows

$$
\begin{aligned}
& L_{0}=\frac{\sinh \beta}{2[\cos (2 \tau)-\cosh (\beta)]} \stackrel{\beta \gg 1}{\simeq}-\frac{1}{2}-e^{-\beta} \cos 2 \tau \\
& L_{x}=\frac{e^{\beta}\left(e^{\beta}-1\right)|\sin (2 \tau)|^{2}}{\left[1+e^{2 \beta}-2 e^{\beta} \cos (2 \tau)\right]^{3 / 2}} \stackrel{\beta \gg 1}{\simeq} e^{-\beta} \sin ^{2} 2 \tau \\
& L_{z}=\frac{e^{\beta}+1}{2\left[1+e^{2 \beta}-2 e^{\beta} \cos (2 \tau)\right]^{1 / 2}} \stackrel{\beta \gg 1}{\simeq} \frac{1}{2}+e^{-\beta} \cos ^{2} \tau,
\end{aligned}
$$

and shows explicitly that optimality of population measurement is recovered for in the low temperature regime $\beta \gg 1$.

\section{CONCLUSIONS}

We have addressed the thermometry of a (directly inaccessible) quantum harmonic oscillator through its coupling to a quantum probe embodied by a controllable qubit that can be subjected to any measurement. By focusing our attention on two models of current physical relevance and using the framework of the (quantum) estimation theory, we have determined the preparation of the probe qubit, the measurement and the value of the interaction time that optimize the estimate of the oscillator's temperature. We found that population measurements performed over the probing system are nearly optimal for an ample range of temperatures. This quite important from the operational point of view, given the handiness of implementing $\hat{\sigma}_{z}$ measurements in all of the settings that have been explicitly addressed here. Our work thus aims at proposing an experimentally viable pathway towards the quantum-limited inference of the properties of inaccessible quantum systems, demonstrating that the paradigm of the coupling with a (fully controllable) low-dimensional quantum system is indeed effective. We are working towards the extension of this framework to explicitly open-system dynamics and the characterization of the environmental properties affecting the dynamics of the harmonic oscillator.

\section{Acknowledgments}

This work has been supported by MIUR (FIRBRBFR10YQ3H-LiCHIS). MP thanks the UK EPSRC for financial support through a Career Acceleration Fellowship (EP/G04759/1) and a grant from the "New Directions for EPSRC Research Leaders" initiative.
[1] S. Campbell, M. Paternostro, S. Bose, and M. S. Kim, Phys. Rev. A 81, 050301 (2010).

[2] L. DiCarlo, M. D. Reed, L. Sun, B. R. Johnson, J. M. Chow, J. M. Gambetta, L. Frunzio, S. M. Girvin, M. H. Devoret, R. J. Schoelkopf, Nature 467, 574 (2010).

[3] K. Hammerer, A. S. Sorensen, and E. S. Polzik, Rev. Mod.
Phys. 82, 1041 (2010).

[4] M. Aspelmeyer, S. Gröblacher, K. Hammerer, and N. Kiesel, J. Opt. Soc. Am. B 27, 189 (2010); F. Marquardt and S. M. Girvin, Physics 2, 40 (2009).

[5] J. M. Raimond, M. Brune, and S. Haroche, Rev. Mod. Phys. 73, 565 (2001). 
[6] D. Hunger, S. Camerer, M. Korppi, A. Jöckel, T. W. Hänsch, P. Treutlein, C. R. Phys. 12, 871 (2011).

[7] M. Paternostro, S. Gigan, M. S. Kim, F. Blaser, H. R. Böhm and M. Aspelmeyer, New J. Phys. 8, 107 (2006); S. Gigan, H. R. Böhm, M. Paternostro, F. Blaser, G. Langer, J. B. Hertzberg, K. C. Schwab, D. Bäuerle, M. Aspelmeyer, A. Zeilinger, Nature 444, 67 (2006).

[8] C. Invernizzi, M. G. A. Paris, S. Pirandola Phys. Rev. A 84, 022334 (2011).

[9] M. Brunelli, S. Olivares, M. G. A. Paris, Phys. Rev. A 84, 032105 (2011).

[10] K. D. B. Higgins, B. W. Lovett, and E. M. Gauger, arXiv:1203.5994 (2012).

[11] B. W. Shore, and P. L. Knight, J. Mod. Opt. 40, 1195 (1993).

[12] C. W. Helstrom, Quantum Detection and Estimation Theory (Academic Press, New York, 1976); A.S. Holevo, Statistical Structure of Quantum Theory, Lect. Not. Phys 61, (Springer, Berlin, 2001).

[13] S. L. Braunstein, C. M. Caves, Phys. Rev. Lett. 723439 (1994); S. L. Braunstein, C. M. Caves, G. J. Milburn, Ann. Phys. 247,
135 (1996).

[14] M. G. A. Paris, Int. J. Quant. Inf. 7, 125 (2009).

[15] S. Stenholm, Phys. Rep. 6, 1 (1973).

[16] Yu. Makhlin, G. Schön, and A. Schnirman, Rev. Mod. Phys. 73, 357 (2001).

[17] K. C. Schwab and M. L. Roukes, Phys. Today 58, 36 (2005); M. Poot, and H. S. J. van der Zant, Physics Reports 511273 (2012).

[18] M. Paternostro, H. Jeong, and M. S. Kim, Phys. Rev. A 73, 012338 (2006); H. Jeong, M. Paternostro, and T. C. Ralph, Phys. Rev. Lett. 102, 060403 (2009); Phys. Rev. A 79, 012101 (2009); G. McKeown, F. L. Semiao, H. Jeong, and M. Paternostro, Phys. Rev. A 82, 022315 (2010).

[19] J. Lee, M. Paternostro, C. Ogden, Y. W. Cheong, S. Bose, and M. S. Kim, New J. Phys. 8, 23 (2006).

[20] The Lambert function of argument $y$ is the inverse function of $y=W e^{W}$, see e.g. R. M. Corless, G. H. Gonnet, D. E. G. Hare, D. J. Jeffrey, and D. E. Knuth, Adv. Comp. Math. 5, 329 (1996). 